\title{
Customer Driven Capacity Setting
}

\author{
Alexander Hübl, Klaus Altendorfer, Herbert Jodlbauer, and Josef Pilstl \\ Upper Austria University of Applied Science, School of Management, \\ Wehrgrabengasse 1-3, 4400 Steyr, Austria \\ \{alexander.huebl, klaus.altendorfer, herbert.jodlbauer, \\ josef.pilstl\}@fh-steyr.at
}

\begin{abstract}
The purpose of this article is to develop a method for short and medium term capacity setting decisions for providing a market oriented level of available capacity for the investigated machine groups. An MTO (make to order) production system is considered. The basic concept is that the cumulative available capacity of the machine group has to be greater than or equal to the cumulative needed capacity influenced by the customer orders. The cumulative needed capacity is corrected with an operation characteristic which defines the slack of the production system, in order to include enough capacity for short term orders.
\end{abstract}

Keywords: Capacity setting, cumulative capacity, Make To Order (MTO), market driven production planning.

\section{Introduction}

Many industries are facing strong global competition where product life cycles are shortened, time to market decreases and customers require fast deliveries of a variety of products of an appropriate quality (see [6]). Therefore it is absolutely necessary that a company ensures that the right product of the right quality is available to the customer in the right quantity at the right time (see [10]). Companies have to adjust their available capacity on customer needs and methods for estimating the needed capacity are crucial for being successful.

If it is not possible to increase the needed capacity then it is essential in terms of customer satisfaction to know what the earliest possible due date is. A high service level can be achieved if due date negotiation is possible, which is according to [14] and [13] a difficult task to perform.

The purpose of this article is to develop a method for short and medium term capacity setting decisions. Reference [7] developed a method where the required customer order lead time and production capacity needed to fulfill customer orders are combined. The basic idea for capacity setting according to [1], [5], [2] as well as [16] is that the cumulative available capacity of the machine group has to be greater than or equal to the cumulative needed capacity for all customer orders which are already in the system. 


\section{Literature Review}

In literature many methods for setting or promising due dates exist, which are directly related to the delivery reliability of orders. In order to promise or set due dates, the available capacity has to be allocated in an MTO environment according to the received customer orders. If the available capacity of the production system is higher than the needed capacity influenced by customer orders, then the difference between the two is wasted capacity (see [4]). But if the available capacity is less than the needed capacity, then the due dates promised to the customer cannot be met. Therefore this paper presents an approach for capacity allocation in an MTO environment.

In [11] a mixed integer programming model with dynamic characteristics is presented for capacity allocation in a supply chain. Moreover heuristics are presented and compared to the results of the mixed integer program in order to demonstrate that the heuristics work well.

Reference [12] developed an analytical model for a capacity allocation problem. The authors assumed a stochastic production capacity and have implemented frequent and occasional customers demanding the capacity. In this paper the expected total income including the penalty costs is maximized. Product mix and sensitivity information allow a guideline for online control systems.

In [17] and [18] a model is published where the production orders are processed by the bill of material (BOM) from the finished goods down to the raw material delivered by the suppliers. At each level of the BOM the inventory is checked if enough material for the orders is available. Missing material is then produced or ordered. This approach supposes deterministic material availability data and no processing times are considered.

Reference [9] developed a mathematical model for a route-independent analysis of available capacity in flexible manufacturing systems whereby the approach is based on the concept of operation types. Moreover a sensitivity analysis is developed to analyze the feasibility of the production system when production requirements and machine capacities changes.

Reference [3] introduced a model which supports decision makers by verifying a customer required due date, whereby potential and already confirmed orders with different probabilities are compared with the actual level of available capacity. A two stage capacity check is applied, where in the first step all resources required by the new order are checked for occurring overloads without the new order. This is followed by a second overload test by including the additional capacity of the new order. Moreover [3] assumed deterministic processing times.

As seen, many methods are available in literature. Reference [11] presented a mixed integer programming model. Reference [12] published an analytical approach for optimizing the total expected income. Reference [9] developed a model for a route-independent capacity analysis. Reference [17] and [18] developed a material based approach. Reference [3] established a model where orders are divided into different probabilities of occurrence.

In our paper the probability of the occurrence of capacity needed based on customer orders depends on the slack between order due date and order date. The difference to [3] is that no explicit future orders are considered but only certain future capacity leads are used for the capacity setting. This means we anticipate future demand also 
with shorter customer required lead time than the orders already being in the production system. As shown in the literature review most of the authors discussing capacity setting assumed a deterministic processing time. Hence, the processing time is supposed to be deterministic in this paper as well.

\section{Model}

At the beginning of this section the concept of operations characteristic (OC) is explained and adapted. This is followed by the development of a capacity allocation model. In the main part of this paper the connection between the concepts of OC and capacity allocation is presented. Finally a short numerical example is given.

\subsection{Model Assumptions}

The model assumes an operation earliest due date (see [15]). Stochastic distributions for interarrival time of customer orders and customer required lead times are assumed. In this model processing times are supposed to be deterministic as argued in the literature review. The machines are clustered into machine groups which fulfill the same processing step.

\subsection{Operations Characteristics}

The concept of applying the operations characteristic (OC) is based on [7], whereby this OC defines the relationship between the customer required lead time and the capacity needed at a machine group. For a one-machine-model the OC shows how much of the customer required capacity is known how many periods in advance. Figure 1 shows such an OC.



Fig. 1. Operations characteristics

The horizontal axis of Figure 1 shows the required customer order lead time. The vertical axis indicates the cumulated workload of the customer orders at the investigated machine group. The example shown in Figure 1 is based on a normally distributed required customer order lead time where the mean is 20 periods and sigma is 10 periods. Five periods before the due date, on average $93.3 \%$ of the required capacity 
of a machine group is known for the production system in the given example. Moreover the integral over OC up to a certain time period returns the average production lead time if all customer orders which have a customer required lead time smaller then the investigated time period (used in the integral) are released to the production system immediately.

In [7], the OC concept encompasses the concept of a constant remaining processing time. This time can be the transportation time to the customer in a onemachine production system or the remaining processing and handling time in a multi machine production system. Since this time is assumed to be constant in [7], which especially for job shop production systems does not hold, the OC concept is slightly changed in this paper. The following definition shown in (1) to (3) is used:

$$
\begin{gathered}
O C_{j}(t)=1-F_{t_{i}(j)}(t) \\
l_{s}(j)=d_{i, j}-o_{i} \\
d_{i, j}=d_{i}-l_{\min }(i, j)
\end{gathered}
$$

Whereby:

- $O C_{j}(t) \ldots$ Operations characteristic, stating what percentage of capacity is, on average, already known $t$ periods before the due date at machine group

- $\quad F_{l(j)}(t) \ldots$ cumulated distribution function of $l_{s}(j)$

- $\quad l_{s}(j) \ldots$ stochastic variable for the slack between machine dependent due date $d_{i, j}$ and order date $o_{i}$

- $\quad d_{i} \ldots$ customer required due date for production order $i$

- $\quad l_{\min }(i, j) \ldots$ minimum technical remaining time to finish the order $i$ after being finished at machine group $j$, includes the processing times of the following processing steps as well as the handling time but no waiting time

The difference between the original concept of [7] and the one applied here is that in [7] the $l_{\text {min }}$ just depends on the machine group but not on the order itself. Especially in cases of job shop production systems with different lot sizes and routings, this additional dependence on the order leads to advantages.

\subsection{Capacity Allocation}

The following section describes the model for capacity setting, which compares the cumulated needed capacity and the cumulated available capacity. The cumulated needed capacity is calculated by summing up the processing and setup times at the machine group $j$ of all orders at their due date as shown in (4) and (5). $A_{j}(t)$ is a non negative monotonically increasing function, which describes how much capacity is needed at the investigated machine to fulfill the customer demand on time. 
The needed capacity of a machine group for producing a final product depends on the bill of material, the routing data, the lot sizes and the standard processing times. Instead of a classical backward scheduling the proposed approach determines the latest possible date for capacity allocation for each machine in the production system by subtracting from the customer confirmed due date only the remaining process-, set up- and transport times (defined as minimum technical remaining lead time $l_{\min }(i, j)$. If a backlog exists at machine group $j$ then $A_{j}(t)$ will not start at zero, because all the late jobs are cumulated into $A_{j}(0)$.

The OC converges asymptotically to the abscissa. Hence, orders with long and extremely long customer required lead times are treated almost identically by the OC correction. Therefore, the work-ahead window $w$, where only those orders are taken into consideration which have a smaller customer required lead time than $w$, is introduced as seen in (5). The work-ahead window is introduced to reduce the finished goods inventory (see [8]) and to set a border for which time frame the short and medium term capacity planning is performed.

$$
\begin{gathered}
A_{j}(t)=\int_{0}^{t} a_{j}(\tau) d \tau+A_{j}(0) \\
a_{j}(t)=\sum_{\substack{d_{i, j}=t \\
d_{i, j} \leq w}} a_{i, j}
\end{gathered}
$$

Whereby

- $\quad A_{j}(t) \ldots$ capacity needed until time $t$ at machine group $j$

- $\quad A_{j}(0) \ldots$ backorder capacity at time 0 at machine group $j$

- $\quad a_{i, j} \ldots$ capacity needed at the machine group $j$ to finish order $i$ (each production order consists of one customer order)

- $\quad a_{j}(t) \ldots$ capacity needed with due date $t$ at machine group $j$

- $\quad w \ldots$ work-ahead window

The cumulated available capacity is calculated by integrating over the planned machine group capacity as seen in (6).

$$
X_{j}(t)=\int_{0}^{t} x_{j}(\tau) d \tau
$$

Whereby

- $\quad x_{j}(t) \ldots$ capacity available at time $t$

- $\quad X_{j}(t) \ldots$ capacity available until time $t$

The model presented in section 3.3 corresponds to the work published in [1] and [5].

\subsection{Model Extension}

By applying the classical approach developed in [1] and [5] no securities for short term capacity allocation exist, because only the capacity for already fixed customer 
orders is included. However, the concept of the operation characteristics developed by [7] and adapted in this paper explains that with a certain probability short term orders, which will decrease over the length of the customer required lead time, will enter the production system. The model developed in this paper combines those two concepts in order to include short term orders.

The problem of implementing enough capacity for short term orders is solved by producing fixed customer orders earlier in order to get free capacity for short term orders. The OC describes exactly how much workload of the orders has to be produced earlier by applying the integral of the OC. Therefore, integrating the OC up to the investigated customer required delivery time indicates how much earlier a capacity for an order has to be provided as seen in (7). This leads to $G_{j}(t)$, which shows the cumulative needed capacity corrected by the OC.

$$
G_{j}(t)=A_{j}\left(\int_{0}^{t} O C_{j}(\tau) d \tau\right)
$$

Whereby $G_{i}(t)$ refers to the corrected capacity needed until time $t$.

To set the capacity in a proper way it is necessary to compare the cumulative available capacity $X_{j}(t)$ provided by the machine group with the corrected cumulative needed capacity $G_{j}(t)$ for producing the customer orders. If $G_{j}(t)$ exceeds $X_{j}(t)$ then orders cannot be produced on time. There are several possibilities for the management to handle this problem, such as, flexible working hours, splitting the lot size or negotiating new due dates for customer orders according to [8]. But, if $X_{j}(t)$ is bigger than $G_{j}(t)$ then the company wastes money because more capacity is provided than needed. In this case it is possible to reduce $x_{j}(t)$ in order to save money.

\subsection{Numerical Example}

A machine group somewhere in the production process is investigated in this numerical example, whereby the customer required due dates are calculated according to (3). In the numerical example $x_{j}(t)$ is assumed to be 3 capacity units per period, whereby preventive maintenance is planned for periods 8 to 10 as illustrated in Figure (2). $A_{j}(t)$ indicates the cumulated needed capacity for all fixed customer orders until time 30, whereby the due date and the required capacity for each order is shown in the table on the right in Figure 2.

Due to the fact that the production system will receive further customer orders according to the OC the system has to provide enough capacity to produce these short term customer orders on time as well. Therefore $G_{j}(t)$ represents the cumulated corrected needed capacity for the investigated machine group, whereby the OC parameters from Figure (1) have been applied. For example the capacity with the due date at time 10 has to be provided until time 9.25, because the integral of the OC from 0 to time 10 is equal to the corrected latest possible date for capacity allocation time. Figure 2 shows the correction of $A_{j}(t)$ which results in $G_{j}(t)$.

If no short term orders are included for capacity planning, then $X_{j}(t)$ is always bigger than $A_{j}(t)$. That means the company is not able to accept orders until time 11 in order to guarantee the due dates negotiated with customers. All orders are fulfilled on time. 


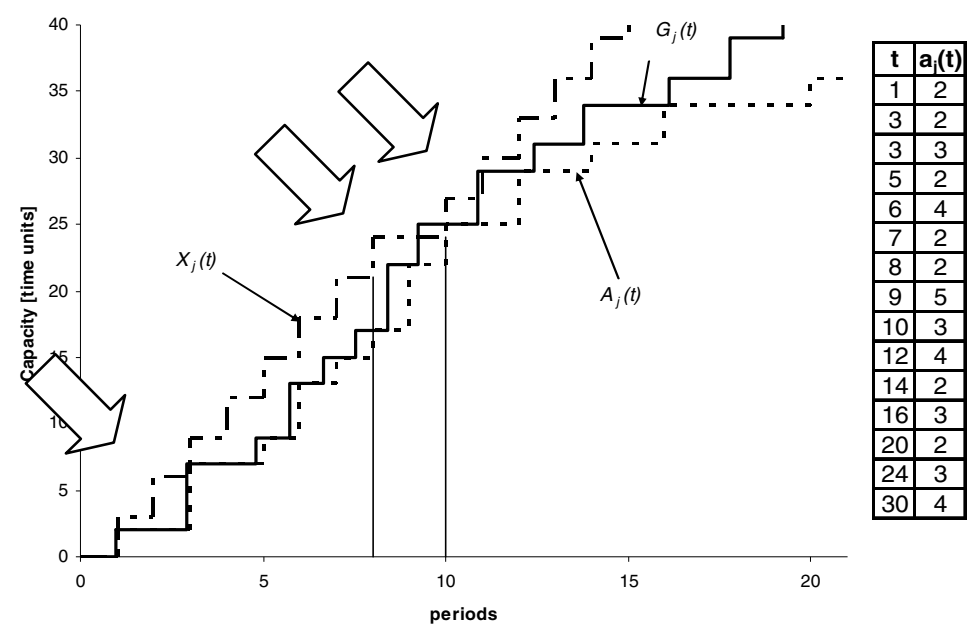

Fig. 2. Capacity checking

Due to the OC it is known that short term orders will enter the system with shorter due dates than the existing orders. Hence capacities are planned earlier than the original due date as seen in Figure 2. After applying the OC the production system cannot produce existing orders or new short term orders on time as shown by the three highlighted crossings of $X_{j}(t)$ and $G_{j}(t)$ in Figure 2. Now actions like flexible working hours, lot size splitting and delaying customer orders can be implemented in advance by the management to increase delivery reliability based on the anticipated future needed capacity.

\section{Conclusion}

In MTO environments it is necessary to allocate production capacity according to customer demand. This paper uses the approach of comparing the cumulated available capacity of the production system and the cumulated needed capacity demanded by the customer. The major drawback of this method is that no capacities for short term orders are included. Therefore, the concept of the operations characteristics, which describes the relationship between customer required lead time and needed capacity, is implemented to calculate how much earlier the needed capacity has to be provided, so that short term orders can be included.

Based on the corrected needed capacity the available capacity of a production system can be adjusted. This can be done by management decisions. If the cumulated available capacity is much higher than the cumulated needed capacity, then reducing the capacity by a reduction of shifts or redundancies can be an option. But if the cumulated needed capacity is higher than the cumulated available capacity the available capacity has to be increased by increasing for example the workforce by personnel leasing otherwise the promised due date cannot be adhered to. Further research should extend this concept to a stochastic processing time. 
Acknowledgments. This paper was written within the framework of the project "Embedded Conwip" funded by the Austrian Research Promotion Agency (FFG).

\section{References}

1. Altendorfer, K., Jodlbauer, H.: An Approach for Capacity Checking and Due Date Adjustment. In: Proceedings FH Science Day 2007 (2007)

2. Altendorfer, K., Jodlbauer, H.: Simple cumulative model for Due Date Setting. In: Leobener Logistic Cases - Supply Network Management (2009) (to appear)

3. Corti, D., Pozzetti, A., Zorzini, M.: A capacity-driven approach to establish reliable due dates in a MTO environment. International Journal of Production Economics 104, 536-554 (2006)

4. Edwards, J.N.: MRP and Kanban - American Style. In: APICS 26th Conference Proceedings, pp. 586-603 (1983)

5. Hübl, A., Jodlbauer, H.: A combined approach for capacity checking, due date setting and stock availability in an MTO environment. In: Proceedings FH Science Day 2008 Linz, Austria, pp. 372-381 (2008)

6. Javalgi, R.G.W., Thomas, W., Ghosh, A.K., Young, R.B.: Market orientation, strategic flexibility, and performance: implications for services providers. Journal of Services Marketing 19(4), 212-221 (2005)

7. Jodlbauer, H.: Customer driven production planning. International Journal of Production Economics 111(2), 793-801 (2008a)

8. Jodlbauer, H.: Produktionsoptimierung - Wertschaffende sowie kundenorientierte Planung und Steuerung, 2nd edn. Springer, Wien (2008b)

9. Koltai, T., Stecke, K.: Route-Independet Analysis of Available Capacity in Flexible manufacturing Systems. Prod. and Operations Management 17, 211-223 (2008)

10. Lambert, D.M., Stock, J.R., Ellram, L.M.: Fundamentals of Logistics Management. McGraw-Hill, New York (1998)

11. Li, H., Hendry, L., Teunter, R.: A strategic capacity allocation model for a complex supply chain: Formulation and solution approach comparison. Int. J. of Prod. Econ. (March 24, 2007) (in Press, Corrected Proof)

12. Modarres, M., Sharifyazdi, M.: Revenue management approach to stochastic capacity allocation problem. Europ. J. of Op. Res. 192(2), 442-459 (2007)

13. Moses, S., Grant, H., Gruenwald, L., Pulat, S.: Real-Time due-date promising by build-toorder environments. Int. J. Prod. Res. 42, 4353-4375 (2004)

14. Özdamar, L., Yazgac, T.: Capacity driven due date settings in make-to-order production systems. Int. J. Prod. Econ. 49, 29-44 (1997)

15. Panwalkar, S.S., Wafik, I.: A Survey of Scheduling rules. Operations Research 25(1), 4561 (1977)

16. Taylor, S., Plenert, G.: Finite Capacity Promising. Production \& Inventory Management Journal 40(3), 50-57 (1999)

17. Xiong, M., Tor, S.B., Khoo, L.P., Chen, C.-H.: A webenhanced dynamic BOM-based available-to-promise system. Int. J. Prod. Econ. 84, 133-147 (2003a)

18. Xiong, M., Tor, S.B., Khoo, L.P.: WebATP: a Web-based flexible available-to-promise computation system. Prod. Plan. \& Control 14, 662-672 (2003b) 